\title{
Approach on Tsallis statistical interpretation of hydrogen-atom by adopting the generalized radial distribution function
}

\section{George Livadiotis}

\section{Erratum to: J Math Chem \\ DOI 10.1007/s10910-009-9524-6}

The original publication contains a spurious 0 at the end of Eq. 12. The equation should appear as follows:

$$
R_{c}(q) \cong 2.741+18.7\left(q-q_{c}\right)
$$

The online version of the original article can be found under doi:10.1007/s10910-009-9524-6.

\section{G. Livadiotis $(\varangle)$}

Section of Astrophysics, Astronomy and Mechanics, Physics Department, National and Capodistrian University of Athens, Panepistimiopolis, Zografos, 15784 Athens, Greece

e-mail: glivad@phys.uoa.gr 